\title{
Risk factors for respiratory disease in chronic psychiatric in patients
}

\author{
Nora Sánchez-Mora, $\mathrm{PhD}^{*}$ \\ Óscar Medina, MD** \\ Beatriz Francisconi, MD*** \\ Néstor W. Meza, PhD* \\ Nineth Rossi, MD* \\ Francisco Colmenares, $M D^{*}$ \\ Mara Parellada Redondo, $\mathrm{PhD}$ ** \\ Celso Arango, $\mathrm{PhD}^{\star *}$
}

* Laboratorio Integrado de la Escuela de Medicina en el Táchira (LABIEMET). Universidad de Los Andes. San Cristóbal, Venezuela

** Departamento de Psiquiatría, Hospital General Universitario Gregorio Marañón, Madrid, Spain

*** Sanatorio Antituberculoso San Cristóbal, Venezuela

VENEZUELA, SPAIN

ABSTRACT - Objective: Individuals with schizophrenia and other chronic mental illnesses present a series of risk factors, that predispose them to extensive medical comorbidity. The aim of this study was to determine the risk factors for respiratory disease in chronic psychiatric inpatients.

Methods: All patients at a long-term mental institution were invited to participate in this study. Socio-demographic data, tobacco and alcohol consumption, respiratory symptoms and chest X-rays were collected from 154 patients. Sputum microscopy and cultures for M. tuberculosis were done in specific cases.

Results: The symptoms reported were cough (58\%); expectoration (44.2\%); dyspnea (28.6\%); hemoptysis $(9.7 \%)$, and chest pain $(20.1 \%)$. There were chest $\mathrm{x}$-ray changes in $57.1 \%$ of patients. These were suggestive of chronic obstructive pulmonary disease (COPD) in $31.2 \%$ and an interstitial pattern in $19.1 \%$. The prevalence of tuberculosis in this sample was $4.5 \%$. Schizophrenia was the most common mental illness $(43.5 \%)$, followed by an organic mental disorder $(32.5 \%)$. We found a positive relationship between schizophrenia and cigarette smoking (odds ratio 2.30, 95\% CI 1.11 to 4.76), and schizophrenia and $x$-ray changes (odds ratio $2.35,95 \%$ CI 1.15 to 4.83 ). There was no significant relationship with other psychiatric disorders. 
Conclusions: The high prevalence of respiratory morbidity in patients with chronic mental illness hospitalized in long-term care facilities, is probably due to factors such as extreme poverty, tobacco consumption, overcrowding, and nutritional deficit.

Received 15 November 2006

Revised 23 April 2007

Accepted 9 May 2007

\section{Introduction}

Individuals with chronic mental disease present a series of general health risk factors, including treatment with a wide range of drugs, substance abuse, poor quality of life, and malnutrition, which predispose them to extensive, but poorly studied medical comorbidity ${ }^{1}$. In addition to the risk factors cited, health care tends to be inadequate, and there is a lack of healthcare policies, aimed at improving the care and quality of life of this largely marginalized and alienated population. The stigmatization of psychiatric patients has often resulted in their exclusion from research programs; justifications for this have included deteriorated cognitive function, psychological regression, and affective deterioration ${ }^{2,3}$. Recent interest has focused on physical illness, especially cardiovascular and endocrine diseases, in patients with schizophrenia ${ }^{4,5}$. However, the assessment of the prevalence of respiratory diseases in patients with chronic mental illness has generally been neglected. The present study was conducted at a long-term care facility in Venezuela with a large population of psychiatric patients, schizophrenia being the most common diagnosis, to assess the prevalence of risk factors for respiratory disease, and determine the prevalence of respiratory conditions in this population.

\section{Materials and methods}

The study's sole inclusion criterion was informed consent for participation. Of the 220 patients with chronic mental disease hospitalized in the facility, 154 were enrolled in the study; the remaining $30 \%$ of the subjects were either excluded due to inability to consent or refusal to participate. We obtained written informed consent from the subjects, the person responsible for the individual, and the medical center's board of physicians. A brief survey was used to collect socio-demographic data (age, sex, origin, and socioeconomic status) using a version of Graffar's method modified for use in Venezuela ${ }^{6}$, and the overcrowding index was also calculated ${ }^{7}$. According to this index, the average space per person should be $4.5 \mathrm{~m}^{2}$. Data were also collected using physical examination and complete questionnaire on respiratory symptoms (cough, expectoration, hemoptysis and dyspnea), which was designed for the study and administered by two respiratory specialists (SMN, FB,) who were not part of the hospital staff. Abuse of or dependence on tobacco, alcohol, and other drugs was also recorded. Tobacco consumption was evaluated as a pack/year index calculated as follows: number of cigarettes smoked per day divided by 20 , multiplied by number of years smoked. Since alcohol was prohibited at the study facility, a retrospective history of alco- 
hol intake before admission was recorded. Patients were categorized either as abstinent or as having a previous history of abuse and/or dependence. Patients' weight and height were measured to calculate their body mass index (BMI). Posterior/anterior chest $\mathrm{X}$-rays were also taken for all patients. Patients with a productive cough had sputum microscopy and cultures for M. tuberculosis.

$\mathrm{X}$-rays were interpreted according to Felson patterns ${ }^{8}$, by two independent observers (respiratory specialists). The two raters, who were blind to subjects' clinical data, scored the chest $\mathrm{x}$-rays independently. The Kappa concordance interobserver index between the two independent raters for the Felson patterns was 0.90 . In the event of differing results, a consensus reading was performed by the two raters. DSM-IV diagnostic criteria $^{9}$, backed up by a previous psychiatric history, were applied to render a psychiatric diagnosis.

The data were analyzed using the EpiInfo statistics software package, version $6.04^{10}$. A Chi-squared test with Yates' correction, corrected with Fisher's exact test if any of the expected values was $<5$, was used to analyze the association of the different risk factors with actual disease. The strength of the relationship between variables and the possible risk of developing a respiratory disease were determined using the odds ratio and confidence intervals with the MantelHaenszel test. To evaluate the interaction between two or more variables that affected the presence of radiological changes in specific mental disorder, we performed a logistic regression models with the variables that in the univariate analyses were significantly related to radiological changes.

The present study was approved by the Ethics Committee of the University of the Andes.

\section{Results}

Socio-demographic. Regarding sociodemographic variables, $57.8 \%$ of the subjects were male and $42.2 \%$ were female, with a mean age of 43.4 years (SD 12.2 years). With respect to origin, $65 \%$ came from rural areas and $35 \%$ from urban areas. No patients were at socioeconomic level I (the wealthiest segment of the population); most patients were at levels IV $(29.2 \%)$ or V $(60.4 \%)$, the latter corresponding to extreme poverty. The mean hospitalization time at the time the study as conducted was 19.3 months, with a standard deviation of 10.2 months (range 3 to 60 months). Patients slept in 4 wards ( 2 for men and 2 for women) with a surface area of $80 \mathrm{~m}^{2}$ each and a mean of 55 patients per ward, i.e., $1.45 \mathrm{~m}^{2}$ per patient, in other words, in seriously overcrowded conditions. The socio-demographic information for the group of patients (30\%) who refused to participate in this study was similar to those who consented; there were no significant differences between the two groups in terms of gender, age, socioeconomic status or psychiatric conditions.

Risk factors. The BMI revealed that no patients were overweight; the majority $(77.3 \%)$ was within normal limits, and the remainder $(22.7 \%)$ was below normal. Examination of clinical respiratory symptom variables in this study revealed persistent cough in $57.8 \%$ of the population, expectoration in $44.2 \%$, dyspnea in $28.6 \%$, hemoptysis in $9.7 \%$, and chest pain in $20.1 \%$ (Table I).

Nearly half of the patients, $48.7 \%$, were current smokers; $10.4 \%$ were ex-smokers, and $40.9 \%$ had never smoked. Tobacco consumption averaged 20 pack-years for the current smokers. There was a statistically significant association between smoking 
Table I

Characteristic of population of study

\begin{tabular}{lcc} 
Characteristic & $\begin{array}{c}\text { Frequency } \\
\mathrm{n}=154\end{array}$ & $\%$ \\
\hline Sexo & & \\
$\quad$ - Male & 89 & 57.8 \\
- Female & 65 & 42.2 \\
\hline Symptoms Clinic & & \\
- Cought & 89 & 57.8 \\
- Expectoration & 68 & 42.2 \\
- Dyspnea & 44 & 9.6 \\
- Hemoptysis & 15 & 20.1 \\
- Pain chest & 31 & \\
\hline Tobacco Consumption & & 48.7 \\
- Current smokers & 75 & 40.4 \\
- Ex-smokers & 16 & 40.9 \\
- Never smokers & 63 & 62.9 \\
\hline Alcohol Consumption & & 37.1 \\
- Ex-drinkers & 97 & 12.4 \\
- Never drinkers & 57 & 87.6 \\
\hline Ilegal Drugs Consumption & & \\
- Use ilegal drugs & 19 & \\
- Never use ilegal drugs & 135 & \\
\hline
\end{tabular}

and male gender (chi-square 22.8, $P<$ 0.001 ), as well as a significant relationship between smoking and cough (chi-square 14.3, $P<0.001$ ). Before admission, 62.9\% were alcohol consumers (including both abuse and dependence), and $12.4 \%$ used illegal drugs, such as marijuana and cocaine. There was a significant relationship between alcohol abuse/dependence and male gender (chi-square 5.5, $P<0.01$ ).

Respiratory disease findings. X-ray changes were present in $57.1 \%$ of the total population, $31.2 \%$ of which were suggestive of chronic obstructive pulmonary disease, characterized by air entrapment with increased pulmonary transparency, enlargement of intercostal spaces, and flattening of the diaphragm; $19.4 \%$ had an interstitial pattern characterized by reticular or nodular reticular infiltration and decreased pul- monary volume; and $6.5 \%$ presented cardiomegaly. There was a positive relationship between $\mathrm{x}$-ray changes and coughing (odds ratio $2.18,95 \%$ CI 1.08 to 4.45 ) and hemoptysis (odds ratio 5.55, 95\% CI 1.13 to 37.04). There was no significant relationship with other respiratory symptoms. When we correlated x-ray changes with the other variables, we found a statistically significant relationship with smoking (odds ratio 4.35, 95\% CI 2.08 to 9.18 ) and with a previous history of alcohol abuse and/or dependence (odds ratio $2.67,95 \%$ CI 1.29 to 5.54 ).

All patients with a productive cough (68/154) were tested for M. Tuberculosis by sputum microscopy and cultures. Tuberculosis was diagnosed during the study in 3 patients (1.9\%) by positive sputum microscopy, confirmed by radiological findings, productive cough, fever, general malaise, and hemopty- 
sis, as well as interstitial infiltration, particularly in the right apical area. There were 4 patients $(2.6 \%)$ in the rehabilitation center who were receiving antitubercular treatment and ongoing medical follow-up at the time the study took place. Therefore, the detected prevalence of tuberculosis in the psychiatric patients participating in the study was $4.5 \%$.

A review of patient records revealed that only $9.7 \%$ had been previously diagnosed with a respiratory disease, such as untreated asthma (3.8\%), tuberculosis for which they were still receiving treatment $(2.6 \%)$, and previously-treated tuberculosis $(3.2 \%)$.

Schizophrenia was the most common mental illness in the sample $(43.5 \%)$, followed by an organic mental disorder $(32.5 \%)$. On correlating different psychiatric diseases with the study variables, we found a positive relationship between schizophrenia and cigarette smoking (odds ratio 2.30, 95\% CI 1.11 to 4.76), and schizophrenia and X-ray changes (odds ratio 2.35, $95 \%$ CI 1.15 to 4.83 ). In the logistic regression model, with psychiatric diagnosis (schizophrenia versus other diagnosis) and smoke habit (smokers and ex-smokers versus never smokers) as independent variables, this last variable significantly predicted radiological changes (Wald $=14.93, P<$ 0.001 , odds ratio $3.95,95 \%$ CI. 1.98-7.92). The diagnosis of schizophrenia did not predict radiological changes when entered in the regression.

\section{Discussion}

In our study group of 154 inpatients hospitalized in a long-term psychiatric unit, we found a high prevalence of $\mathrm{x}$-ray changes involving more than half of the study popu- lation $(57.1 \%)$. These findings are higher than those reported in previous studies ${ }^{11}$ and even more remarkable when compared to the published data from public health studies $^{12}$. We have not found published studies on respiratory disease in the general population of Venezuela to compare with our results, but we would expect a much lower prevalences of $\mathrm{x}$-ray changes in the general population.

Studies focusing on morbidity in patients with chronic mental illness have reported a high prevalence of certain physical diseases in these patients ${ }^{1,13}$. Previous studies have found a high prevalence of respiratory, cardiovascular, and endocrine diseases ${ }^{11,13}$. Respiratory disease in patients with chronic mental illness had not previously been documented in Venezuela and there are few epidemiological studies in the literature.

Based on our research, we can see that community psychiatric practice poses an additional complication and one that is rarely acknowledged, i.e., that these patients are deprived of necessary medical care, so that they are more likely to suffer from severe, undetected physical diseases ${ }^{14}$. In our study, we found that the percentage of patients with a prior diagnosis of respiratory disease was low $(9.7 \%)$ in light of the risk factors in these patients, combined with the high percentages of $\mathrm{x}$-ray changes and respiratory symptoms observed after hospitalization.

Possible factors raising the morbiditymortality index in the study population include unhealthy lifestyles, particularly cigarette-smoking, as well as lack of recognition and treatment of physical diseases $^{1,15,16}$.

Another study conducted at the same psychiatric rehabilitation center ${ }^{17}$ found that the population had scant economic resources 
and a monotonous diet of 2,000 $\mathrm{kcal} / \mathrm{day}$, resulting in patients with a subnormal body mass index, which, in addition to the overcrowded patient conditions, predisposes the population to a wide variety of physical diseases. Any nutritional deficiency, whether due to reduced caloric intake, loss of essential nutrients, or changes in intermediate metabolism, may lead to lowered cell-mediated immunity ${ }^{18}$, which may in turn increase the prevalence of tuberculosis.

In our study population, $89.6 \%$ were living in poverty (Graffar's levels IV and V). This is higher than the level in the general population in the country where the study was conducted, in which a $79.8 \%$ of the population are at levels IV and $\mathrm{V}^{19}$. In most Venezuelan hospitals, economic resources are insufficient to provide patients with optimal health care. In general, patients are obliged to cover the costs of their own medical treatment, laboratory tests, and x-rays. The center in our study is not exempt from this national phenomenon, and therefore we assume that our results are representative of other long-term psychiatric facilities in that country. When added to the frequent abandonment of such patients by their families, this results in a lack of access to the proper treatment for their mental and physical diseases.

The published studies on psychiatric patients with respiratory disease are based on symptom scales. Our study used the presence of symptoms supported by radiological findings, showing a high prevalence of clinical respiratory symptoms, particularly cough with expectoration and $\mathrm{x}$-ray changes suggestive of chronic obstructive pulmonary disease. Diagnosis of tuberculosis in patients with clinical respiratory symptoms in Venezuela is based primarily on sputum microscopy and culture. The state of Tachira, where the study was con- ducted, may be considered an endemic tuberculosis area (the national incidence rate in 2000 was 25.2 new cases per 100,000 habitants ${ }^{20}$, where the Mantoux test has low specificity as a diagnostic tool, not only due to the population's exposure to the bacillus, but also to BCG vaccination at birth. Sputum studies in the clinical cases of productive cough revealed 3 new cases, resulting in a prevalence of $4.5 \%$. Given the overcrowded conditions in which these patients live, this may be considered a permanent source of contagion.

Cigarette smoking is a risk factor for respiratory disease and also for a variety of illnesses including cardiovascular disease and different cancers. In our study, we found a large number of current heavy smokers $(48.7 \%)$. This level is higher than the $30 \%$ prevalence among adults in the general population in Venezuela ${ }^{20}$ as the $28 \%$ prevalence in San Cristobal city capital of Táchira state. However, the prevalence by sex in Táchira $($ male $=53 \%$; female $=47 \%$ ) is similar to the results in our study ${ }^{21}$. The most common reason given by ex-smokers for quitting was that their families could not afford to buy them cigarettes. This habit was associated with males, who had a higher prevalence of respiratory problems and with the diagnosis of schizophrenia; these results are consistent with the literature ${ }^{22-24}$. However, Osborne et al. ${ }^{25}$ have shown that individuals with schizophrenia living in the community accept health screening and advice to give up smoking as part of a cardiovascular risk reduction initiative. To our knowledge, no studies have examined smoking cessation programs for inpatients with chronic schizophrenia. Progress will depend on both mental health and primary care staff being aware of the problem and being willing to find solutions acceptable and useful to patients, such as improving 
nutrition, implementing educational programs, smoking cessation initiatives or at least limiting smoking areas.

Our results show that there is a high prevalence of undetected respiratory disease in patients with chronic mental illness hospitalized in long-term care centers, very likely due in part to conditions there that promote prevalence. There is an imperative need for a multidisciplinary approach to the medical care provided to patients in such centers, in order to facilitate mechanisms that promote early diagnosis and appropriate treatment, thereby decreasing morbidity from these and other physical diseases ${ }^{26}$. Hospital conditions may be improved through preventive action, such as decreasing overcrowding, controlling tobacco consumption, and improving screening for respiratory risk factors.

\section{Funding and support}

This study was supported by the Ministry of Science and Technology-FONACIT, Venezuela, grant S1-2000000669.

\section{Acknowledgements}

The authors wish to thank Dr. Antonio Arellano, Dr. Carlos Contreras and Dr. Diana Molina.

\section{References}

1. Jeste DV, Gladsjo JA, Lindamer LA, Lacro JP. Medical comorbidity in schizophrenia. Schizophr Bull 1996; 22(3): 413-430.
2. Ramsay RA, Krakowski AJ, Rydzynski Z, Jarosz M, Engelsmann F, Ananth J. International comparison of the prevalence of psychosomatic disorders in schizophrenic patients. Psychother Psychosom 1982; 38(1): 206-220.

3. Haghighat R. The Development of an Instrument to Measure Stigmatization: factor analysis and origin of stigmatization. Eur J Psychiat 2005; 19(3): 144-154.

4. Hennekens CH, Hennekens AR, Hollar D, Casey DE. Schizophrenia and increased risks of cardiovascular disease. Am Heart J 2005; 150(6): 1115-1121.

5. Meyer J, Koro CE, L'Italien GJ. The metabolic syndrome and schizophrenia: a review. Int Rev Psychiatry. 2005; 17(3): 173-180.

6. Méndez CH, Méndez MC. Estratificación social y biología humana. Método Graffar modificado. Archivos Venezolanos de Puericultura y Pediatria 1986; 49(3-4): 93-101.

7. Larralde C, Padilla A, Hernandez M, Govezensky T, Sciutto E, Gutierrez G, et al. [Seroepidemiology of cysticercosis in Mexico]. Salud Publica Mex 1992; 34(2): 197-210.

8. Felson B, Weinstein A, Spitz H. Principles of Chest Roentgenology: A Programmed Text. $1^{\text {a }}$ edn. Spain: Scientific-Medical; 1985.

9. American Psychiatric Association. Diagnostic and Statistical Manual of Mental Disorders: DSM-IV. Washington DC: American Psychiatric Association; 1994.

10. Dean AG, Dean JA, Burton AH, Dicker RC. EpiInfo, version 6,04: a word processing, datebase and statistics program for epidemiology on microcomputers. Atlanta: Centers for Disease Control. 1990.

11. Kendrick T. Cardiovascular and respiratory risk factors and symptoms among general practice patients with longterm mental illness. Br J Psychiatry 1996; 169(6): 733-739.

12. Bennett N, Dodd T, Flatey J. Health survey for England, London: Her magesty's stationery office. 1994.

13. Dickey B, Normand SL, Weiss RD, Drake RE, Azeni H. Medical morbidity, mental illness, and substance use disorders. Psychiatr Serv 2002; 53(7): 861-867.

14. Honig A, Pop P, Tan ES, Philipsen H, Romme MA. Physical illness in chronic psychiatric patients from a community psychiatric unit. The implications for daily practice. Br J Psychiatry 1989; 155: 58-64.

15. Brown S, Birtwistle J, Roe L, Thompson C. The unhealthy lifestyle of people with schizophrenia Psychol Med. 1999; 29(3): 697-701.

16. McCreadie RG, MacDonald E, Blacklock C. Dietary intake of schizophrenic patients in Nithsdale, Scotland, case control study. British Medical Journal 1998; 317: 784-785. 
17. Baptista T, Lacruz A, Meza T, Contreras Q, Delgado C, Mejias MA, et al. Antipsychotic drugs and obesity: is prolactin involved? Can J Psychiatry 2001; 46(9): 829-834.

18. Chandra RK, Kumari S. Nutrition and immunity: an overview. J Nutr 1994; 124(8 Suppl): 1433S-1435S.

19. Landaeta M, Fossi M, Cipriani M, Busto K, Garcia $\mathrm{K}$, Escalona J, et al. El hambre y la salud integral. An Venez Nutr 2002; 16(2):105-111.

20. Pan American Health Organization. Venezuela: Health situation analysis and trends summary. 2005. Avalaible at: http://www.paho.org/English/DD/AIS/cp_862.htm.

21. Briceño E, Angeles M, Sanguino B, Cuesta F. Epidemiología del tabaquismo en la Ciudad de San Cristóbal Estado Táchira. Col Med Edo Tachira 1993; 2(1): 4-10.

22. de Leon J, Diaz FJ. A meta-analysis of worldwide studies demonstrates an association between schizophrenia and tobacco smoking behaviors. Schizophr Res 2005; 76(2-3): 135-157.

23. Glassman AH. Cigarette smoking: implications for psychiatric illness. Am J Psychiatry 1993; 150(4): 546-553.
24. Hughes JR, Hatsukami DK, Mitchell JE, Dahlgren LA. Prevalence of smoking among psychiatric outpatients. Am J Psychiatry 1986; 143(8): 993-997.

25. Osborn DP, King MB, Nazareth I. Participation in screening for cardiovascular risk by people with schizophrenia or similar mental illnesses: cross sectional study in general practice. Bmj 2003; 326(7399): 1122-1123.

26. Lyketsos C, Huyse F, Gitlin D, Levenson J. Psychosomatic medicine: A new psychiatric subspecialty in the U.S. focused on the interface between psychiatry and medicine. Eur J Psychiat 2006; 20(3): 165-171.

\author{
Address for correspondence: \\ Nora Sánchez-Mora \\ Hospital General Universitario Gregorio Marañón \\ Departamento de Psiquiatría, Despacho 18, PB \\ C/ Ibiza 43, 28009 Madrid \\ Spain \\ Telephone: 0034-915-868133 \\ Fax: 0034-914-265005 \\ nora.sanchez@urjc.es - omedina@hggm.es
}

